Revue scientifique sur la conception et l'aménagement de l'espace

$8 \mid 2012$

Les concepteurs de jardins et de parcs japonais

\title{
Du socle au paysage : essai pour un nouveau regard sur les reliefs
}

From bedrock to landscape: an essay for a new perception of landforms

\section{Claire Portal}

\section{OpenEdition}

\section{Journals}

Édition électronique

URL : http://journals.openedition.org/paysage/15863

DOI : $10.4000 /$ paysage. 15863

ISSN : 1969-6124

\section{Éditeur :}

École nationale supérieure du paysage de Versailles-Marseille, Institut national des sciences appliquées Centre Val de Loire - École de la nature et du paysage, École nationale supérieure d'architecture et de paysage de Bordeaux, École nationale supérieure d'architecture et de paysage de Lille, Agrocampus Angers

\section{Référence électronique}

Claire Portal, «Du socle au paysage : essai pour un nouveau regard sur les reliefs », Projets de paysage [En ligne], 8| 2012, mis en ligne le 13 juillet 2012, consulté le 11 mars 2021. URL : http:// journals.openedition.org/paysage/15863; DOI : https://doi.org/10.4000/paysage.15863

Ce document a été généré automatiquement le 11 mars 2021.

Projets de paysage 


\section{Du socle au paysage : essai pour un nouveau regard sur les reliefs}

From bedrock to landscape: an essay for a new perception of landforms

\section{Claire Portal}

Cet article synthétise une étude effectuée dans le cadre d'une thèse de géographie (Portal, 2010) et s'inscrit dans un champ de recherche émergent associé à la géomorphologie culturelle. Cette discipline « étudie la composante géomorphologique d'un territoire comme élément de culture du paysage et pour ses interactions avec les biens culturels de types archéologiques, historiques, architecturaux, etc. " (Panizza et Piacente, 2003, p. 2). La première partie de cette définition fait l'objet d'un approfondissement conceptuel sur la dimension artistique et esthétique des reliefs qui sont considérés comme un motif de paysage, soit en tant qu' «élément de l'espace concret qui nous motive à inventer un paysage " (Aubry, 2006, p. 68). La réflexion menée ici propose un nouveau regard qui s'inscrit à la suite de l'analyse menée par Jean-Pierre Nardy (1982) sur l'évolution de la perception des reliefs par les arts et la sculpture. Ainsi, traditionnellement perçus par une géomorphologie naturaliste depuis la fin du $\mathrm{XIX}^{\mathrm{e}}$ siècle, les reliefs constituent l'armature du paysage "physique " (le "socle géographique », naturel, permanent et spécifique du paysagiste, Davodeau, 2008) mais aussi du paysage sensible et culturel (Portal, 2010). Alors que les reliefs deviennent un motif de paysage au même titre que d'autres éléments, le socle du géographe-géomorphologue devient culturel. Dans ce contexte, cet article affiche trois objectifs principaux :

- Tout d'abord, il porte un intérêt à la description et à la nomenclature précise des reliefs ${ }^{1}$, « la formulation juste et précise des traits du relief étant une mission de base des paysagistes » (Rumelhart, 2000, p.167). Un oronyme indique par sa seule terminologie le processus entraînant la genèse de la forme.

- Ensuite, pour chacun des reliefs et des modelés étudiés, les principaux fondements artistiques vecteurs de leur artialisation (Roger, 1995) sont indiqués, en référence à Simon Schama (1999) et Nadeije Laneyrie-Dagen (2008) qui montrent par leurs écrits que le « roc» ou la «terre » sont des éléments incontournables dans la représentation des paysages. Ce 
nouveau regard intègre les reliefs dans l'espace géographique global (vu, perçu et représenté) et les place en "situation iconique" (Monnier, 1984. p. 235) qui interagit avec les autres éléments composant le paysage artialisé. Les reliefs sont étudiés par une approche iconographique (cartes postales anciennes, peintures, dessins, bandes dessinées, cinéma, publicité, etc.), par des écrits littéraires (voyages pittoresques, récits journalistiques, guides, légendes) ainsi que par leurs fonctionnalités (habitat, agrément, etc.), en association avec d'autres éléments du paysage (héritage archéologique, place forte, site religieux, etc.) (Portal, 2010). Ils sont ainsi intégrés à la construction des modèles paysagers, à la charnière entre art et science.

- Cette imbrication art/science - esthétique/géomorphologie - a notamment été évoquée par Georges Farhat (2008) lors de son étude sur la perspective topographique autour de l'œuvre d'André Le Nôtre et par Alain Freytet et Marc Rumelhart (2010) à propos du blocdiagramme. Elle inscrit cette démarche au cœur conceptuel du diptyque culturaliste et phénoménologique "socle-motif». Cette approche propose de mettre en regard le socle physique $^{2}$ du paysagiste avec la perception culturelle du géomorphologue-géographe, révélant «l'identité d'un paysage - sa singularité - [qui] mêle le socle géographique sur lequel se déroule toute action humaine, l'entité politique et administrative dans laquelle on agit, et le rapport culturel que les hommes entretiennent à leur environnement » (Chomarat-Ruiz, 2010).

\section{Reliefs et modelés : des motifs paysagers du « socle »}

2 En s'appuyant sur la figure 1, la première partie de cet article décline brièvement les principaux regards portés sur les reliefs et modelés. Cette grille résulte d'une l'analyse menée au cours d'une thèse de doctorat (Portal, 2010, op.cit.) et s'appuie principalement sur des sources iconographiques, parfois rehaussées de références littéraires qui se rattachent au relief évoqué. Elle recoupe les perceptions du géomorphologue naturaliste avec les principales fonctionnalités des reliefs (habitat, militaire, agrément, ressource, spirituel) et leur influence dans l'esthétisation des paysages (les reliefsmotifs). Cette première figure décline les grands types de formes qui sont évoquées ainsi que les principales fonctionnalités qui y sont associées et leur influence dans la création d'une relation entre reliefs et sociétés; elle montre finalement que la perception culturelle des reliefs est toujours en évolution. 
Figure 1. Les formes du relief : typologie, motif de paysage et esthétisation des paysages géomorphologiques

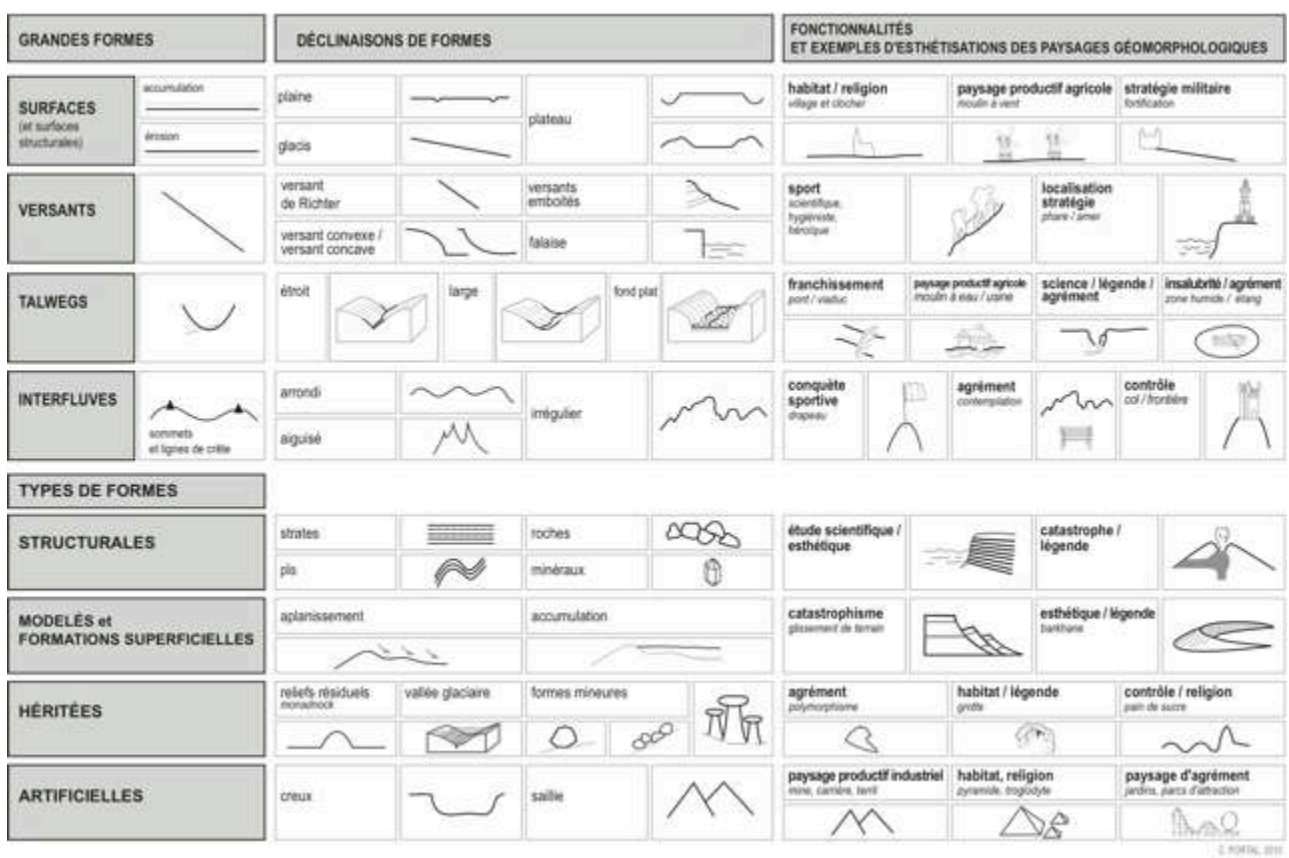

Fonctionnalités et influences des reliefs dans l'artialisation des paysages.

Source : extrait de la thèse de Claire Portal, 2010).

\section{Surfaces, versants, talwegs et interfluves : l'armature des paysages}

\section{Les surfaces et les « pays plats »}

Une surface est une étendue terrestre relativement plane. Elle peut être d'origine structurale ou associée à l'érosion. La genèse de leur planéité est issue soit de l'ablation (surfaces d'érosion) soit de l'accumulation de matériaux. En termes topographiques, les surfaces prennent la forme de plaines, de plateaux et de glacis. L'expression "plat pays » est souvent utilisée pour qualifier ces grandes étendues de terre ou d'eau. Dans les régions d'Europe occidentale, les surfaces sont polysémiques. Synonymes de terres agricoles, elles symbolisent la ruralité, la campagne productive et industrielle; inversement, l'homogénéité des grandes plaines agricoles leur confère ennui et morosité que certaines références littéraires bien connues illustrent ${ }^{3}$. Sur une plaine ou un glacis, tout est visible et donc exposé. N'importe quel artefact vertical situé sur une surface prédomine dans le paysage car reposant sur une armature sans aspérités. Le moindre relief résiduel prend une importance capitale : il est utilisé comme point de vue stratégique ou comme site religieux. Si la surface ne présente aucune aspérité naturelle, le relief manquant est recréé.

Les cathédrales prendront allure de montagnes, les clochers et les moulins à vent, d'amer. 
Figure 2. Jacques Brel, Le Plat Pays, 1962

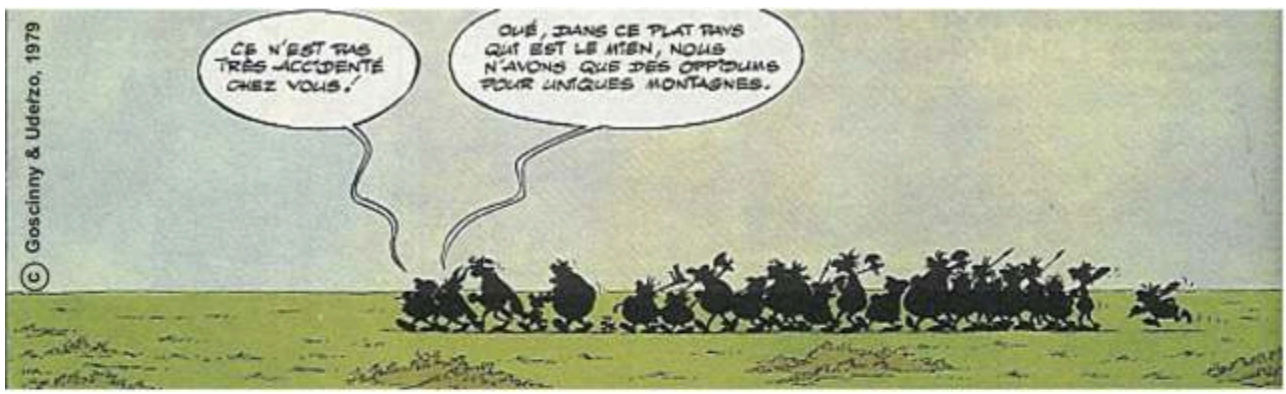

Avec la mer du Nord pour dernier terrain vague

Et avec des vagues de dunes pour arrêter les vagues

Et de vagues rochers que les marées dépassent

Et qui ont à jamais le coeur à marée basse

Avec infiniment de brumes à venir

Avec le vent d'est écoutez-le tenir

Le plat pays qui est le mien

Avec des cathédrales pour uniques montagnes

Et de noirs clochers comme mâts de cocagne

Oủ des diables en pierre décrochent les nuages Avec le fil des jours pour unique voyage

Et des chemins de pluies pour unique bonsoir Avec le vent d'ouest écoutez-le vouloir Le plat pays qui est le mien
Avec un ciel si bas qu'un canal s'est perdu Avec un ciel si bas qu'il fait l'humilité Avec un ciel si gris qu'un canal s'est pendu Avec un ciel si gris qu'il faut lui pardonner Avec le vent du nord qui vient s'écarteler Avec le vent du nord écoutez-le craquer Le plat pays qui est le mien

Avec de l'Italie qui descendrait l'Escaut Avec Frida la Blonde quand elle devient Margot Quand les fils de novembre nous reviennent en ma Quand la plaine est fumante et tremble sous juillet Quand le vent est au rire quand le vent est au blé

Quand le vent est au sud ècoutez-le chanter Le plat pays qui est le mien.

La chanson évoque le paysage de Flandres, pays natal de l'auteur, qui n'est doté d'aucun relief montagneux. On retrouve des allusions à ce plat pays dans d'autres types de littérature : dans la bande dessinée Astérix chez les Belges (Goscinny et Uderzo, 1979), les gaulois font un commentaire sur le paysage qui n'est pas très accidenté et au chef belge de répondre: «Dans ce plat pays qui est le mien, nous n'avons que des oppidums pour uniques montagnes ».

\section{Les versants : de falaises en coteaux}

Un versant est un relief incliné. Il constitue les flancs d'une vallée, d'une colline et le littoral rocheux du continent (falaise). Il se caractérise par sa déclivité, sa hauteur (dénivelé), son profil linéaire ou accidenté et son exposition. Les types de profil de versants introduisent une grande variété de représentations picturales et littéraires, associée, entre autres, à la pratique sportive et contemplative. Les falaises littorales constituent des versants spécifiques : elles dévoilent la structure interne du continent et leurs points culminants - vus de la mer - sont souvent utilisés comme amers, elles sont parfois rehaussées de phares ou de forteresses. Vues du continent, les falaises constituent le mirador de la mer, particulièrement prisé des peintres et des écrivains romantiques (voir figure suivante : http://www.photo.rmn.fr/cf/htm/cSearchz.aspx? $\mathrm{o}=\&$ Total=2\&FP=68563358\&E=2K1KTSGWBUW47\&SID=2K1KTSGWBUW47\&New=T\&Pic=2\&SubE=2C6NU0HUOR59).

\section{Les talwegs et les marais}

6 Le talweg correspond à une ligne joignant les points les plus bas d'une vallée dont la forme est en partie en fonction des processus d'érosion (fluviale, "vallée en V »glaciaire, "vallée en auge ou en $U$ »). Les talwegs sont le plus souvent longilignes, mais dans des cas de dépressions du sol, ils peuvent être recouverts d'une surface en eau (zones humides). La plaine alluviale correspond à un vaste talweg, avec des versants peu inclinés et peu marqués; à l'inverse, une vallée peut être encaissée avec des versants abrupts et un talweg étroit (gorge). Si le talweg est représenté pour lui-même dans certaines œuvres picturales du XIX siècle, il constitue le plus fréquemment un 
décor œuvrant à la mise en scène d'un autre élément du paysage (pont, moulin, embarcation) ou d'un personnage.

\section{Les interfluves et les sommets}

7 Un interfluve est un relief qui sépare deux bassins-versants. Il correspond aux points les plus hauts d'un versant et donc d'une vallée. Ses points culminants, les sommets, sont peu perceptibles en pays de plaine, mais extrêmement découpés en montagne (ligne de crête). Dans les "pays plats", les interfluves se présentent sous forme linéaire, sans réelles aspérités. Ils sont davantage visibles sur une carte topographique par l'intermédiaire des points cotés que sur le terrain. Dans un relief de basses terres, les sommets forment des lignes de partage des eaux vallonnées, constituées de collines dont l'altitude peut varier de 100 à 300 mètres (George et Verger, 2006, p. 80). Ces collines peuvent être isolées les unes des autres ou constituer un soubassement ondulé délimitant des bassins-versants contigus. En montagne, les sommets des interfluves se déclinent selon différents profils : ils se présentent sous la forme de pic, d'aiguille ou de dôme et caractérisent ainsi le faciès des lignes de crêtes.

\section{Structure, modelés, héritages : reliefs-motifs et esthétiques géographiques}

8 Les principaux reliefs qui arment les paysages occidentaux constituent des motifs majeurs, symboles de stabilité et de permanence. Ils supportent le déroulement les récits littéraires ou picturaux. D’autres reliefs/modelés, souvent détaillés à une échelle plus fine, ajoutent une dimension esthétique à la narration.

\section{La structure : « du peintre-artiste au peintre-géologue »}

9 La structure du relief est déterminée par sa géologie (nature et agencement de volumes rocheux). De cette structure interne va dépendre la résistance des roches à l'érosion, impliquant des faciès variés et donc des représentations artistiques diverses. Certains artistes ont joué avec l'esthétique des strates sédimentaires ${ }^{4}$ et d'autres avec les couleurs des roches : dans l'art byzantin et dans la peinture italienne, les décors arides et rocailleux ne sont nullement une imitation de la nature mais ont une "vocation scénographique et allégorique en tant que témoins de la condition de l'homme après le péché originel» (Laneyrie-Dagen, 2008, p. 86). La structure est ici une "poétique de l'espace " (ibid., p. 93) et non une connaissance géologique. La représentation des roches et des minéraux connaît un nouvel essor à la Renaissance qui inscrit le minéral dans le cycle historique de l'Univers, soit dans une durée où se succèdent constructions et destructions ${ }^{5}$. Ce souci de la précision scientifique associée à l'esthétique se retrouve chez les explorateurs comme Alexander von Humboldt (Debarbieux, 2008), par la représentation des volcans ${ }^{6}$ notamment.

\section{Les modelés et les formations superficielles}

10 Les modelés correspondent à l'ensemble des formes de la surface terrestre dont la définition fait appel à un agent ou à un processus d'érosion (plages, dunes et éboulements sont des modelés d'accumulation). Finalement, le modelé désigne la forme des formes du relief (leur faciès). De la structure et des agents d'érosion dépendent les 
profils des surfaces, des versants, des interfluves et des talwegs ${ }^{7}$. Les modelés de versants et leurs représentations sont multiples : l'alpiniste hérö̈que se construit par une ascension souvent expiatoire vers le sommet et s'accomplit dans une souffrance sportive et/ou spirituelle (l'ascension du mont Ventoux par Pétrarque, Besse, 2000). Dans cette sublimation de la nature, les éboulements sont fréquemment représentés en lien avec une catastrophe ${ }^{8}$. Dans un autre registre, les grandes dunes terrestres sont de véritables montagnes mobiles inspirant nombre de récits de voyages (Marco Polo, Guy de Maupassant, Charles Darwin, etc.) et des légendes locales (le " chant des dunes »). Vues du ciel, ces dunes créent des formes courbes et linéaires, prisées des photographes (La caravane de dromadaire dans les dunes près de Nouakchott, Mauritanie, Yann ArthusBertrand, 1999).

\section{Les traces des processus d'érosion : les « héritages naturels »}

Les modelés et les formations superficielles, lorsqu'ils se situent dans des domaines de biostasie, ne constituent plus des formes vives ${ }^{9}$. Ce sont des paléoformes qui sont des " héritages naturels" (les vallées glaciaires dans les milieux tempérés par exemple). Parmi ces héritages, la pénéplaine et les formes résiduelles qui ont échappé à l'aplanissement constituent des reliefs-motifs associés à des pratiques naturalistes et spirituelles. Le géomorphologue William Morris Davis a nommé monadnocks ces reliefs isolés, du nom d'un mont qui domine le bouclier canadien en Nouvelle-Angleterre. Les monadnocks sont souvent des points de vue uniques à des kilomètres à la ronde et s'inscrivent à l'interface entre images réelles, souvent naturalistes, et représentations virtuelles, divines et transcendentalistes (Portal, 2010, p. 81). 
Figure 3. Le mont Monadnock est avec ses 965 mètres le plus haut point dans les 50 kilomètres alentours

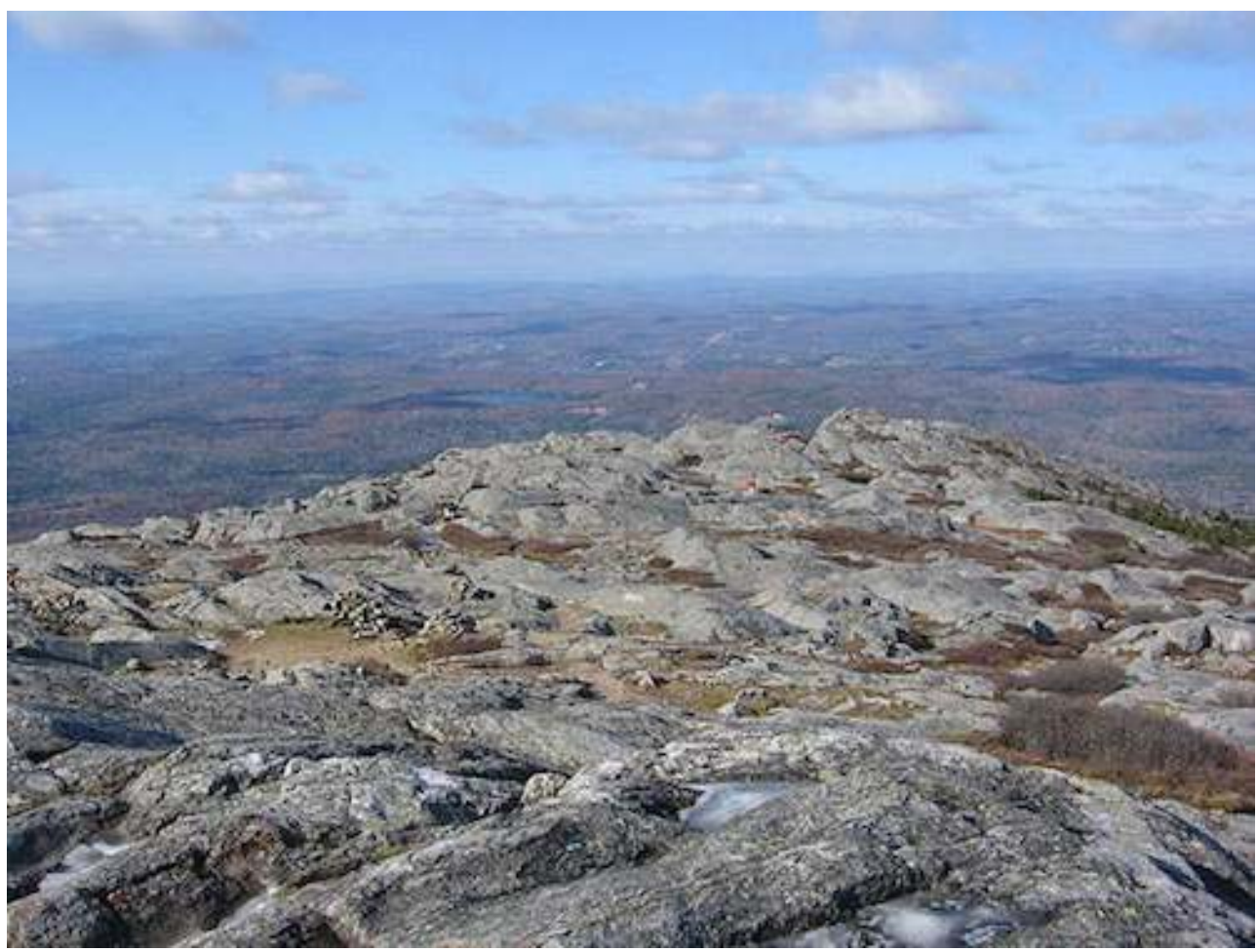

Le Gran Monadncok surplombe la pénéplaine de 600 mètres environ. À la fin du xıx siècle, Ralph Waldo Emerson lui consacre un poème ; Henri David Thoreau est considéré comme le père du premier inventaire naturaliste sérieux de la montagne (Brandon, 2007).

Il en est de même pour les cavités souterraines héritées de l'érosion hydrologique. Celles-ci se situent au pied des falaises littorales et dans des milieux continentaux le plus souvent karstiques. Une terminologie variée désigne ces cavités, associée à des significations spécifiques : l'«antre » symbolise la profondeur, l'essence même de la montagne ; le " gouffre » représente la verticalité, l'insondabilité et l'effroi (Rey, 1992, p. 1613); "grotte» et «caverne» sont synonymes d'habitat, d'abri («biblique», préhistorique, troglodytique) et de réflexions philosophiques (Platon et l'allégorie de la caverne ${ }^{10}$ ). Comme le montre Mélanie Duval (2007) et Nadeije Laneyrie-Dagen (2008), ces cavités ont attiré la curiosité des savants de la Renaissance mais leur méconnaissance les a souvent amenés à dépasser la science pour des considérations plus mystiques.

Reliefs et modelés, petites et grandes formes participent ainsi à la composition paysagère et constituent des motifs de paysage à l'interface entre regards naturaliste et culturel. Ainsi, si le relief-motif constitue bien un élément de l'espace concret motivant l'invention paysagère artistique (Aubry, op.cit.), il est aussi une matière " plastique » à laquelle sens et valeurs sont donnés (Freytet et Chazelle, 2008, p. 24). Si les formes du terrain peuvent être considérées comme " une charpente sur lesquels s'enracinent les autres motifs » du paysage (ibid., p. 64), le relief constitue un motif en lui-même, parfois emblématique (focalisant l'intérêt principal du paysage, le monadnock par exemple) et parfois de détails - comme les strates géologiques de Van Eyck ou de Monet -, qui "sans être indispensable, confère aux sites et aux lieux une ambiance particulière " (ibid., p. 25). La plaine et son horizon constituent quant à eux les archétypes du « relief- 
charpente" où se déroule la scène, attribuant son identité au paysage. Les représentations des reliefs et des modelés interviennent de ce fait dans la construction des modèles paysagers: le socle du paysagiste, essentiellement perçu comme un élément naturel et physique du paysage, accède ainsi à un nouveau regard culturel.

\section{Du socle-motif aux modèles paysagers}

14 Les modèles paysagers se définissent comme des "schèmes culturels structurant les représentations sociales du paysage » (Luginbühl, 2006, p. 64). Plusieurs modèles paysagers se succèdent au fil des époques : les modèles bucoliques et pastoraux sont les premiers à apparaitre et dominent la pensée du paysage pendant la Renaissance. Le modèle sublime « formalise la découverte par l'élite sociale d'une beauté singulière des espaces de grande nature (montagnes, rivages, déserts, essentiellement) (ibid., p. 65). L'essor de la mobilité touristique au XVIII ${ }^{\mathrm{e}}$ siècle et surtout au XIX ${ }^{\mathrm{e}}$ siècle fait émerger et diffuse le modèle pittoresque, dont les marques sont visibles dans l'élaboration des lois de protection du paysage au tournant du $\mathrm{xx}^{\mathrm{e}}$ siècle. Dans chacun de ces modèles, reliefs et modelés constituent des fils conducteurs à l'histoire, tantôt en tant que décor scénographique diffuseur d'ambiances, tantôt comme élément principal participant à la construction des modèles paysagers.

\section{Des reliefs sublimes aux décors fantastiques}

15 Le modèle paysager associé au sublime s'inscrit dans la conception divine et surnaturelle de la «nature »: sur le littoral, l'homme est au cœur de la tempête; en montagne, il se fait minuscule, écrasé par des reliefs exacerbés. La conquête des points culminants est d'abord une expression du sentiment divin par la domination des sommets. Du sublime aux représentations fantastiques, les reliefs s'insèrent dans trois conceptions majeures: l'une est véhiculée par les membres des clubs alpins au $\mathrm{XIX}^{\mathrm{e}}$ siècle où seuls la dangerosité de l'expérience et l'effort intense permettent de saisir la « vérité » des montagnes (Schama, 1999, p. 557). A contrario, la vision de «l'alpiniste sentimental » considère que l'expérience authentique de la montagne n'est possible que par la contemplation ${ }^{11}$. Ainsi, pour John Ruskin (artiste et critique d'art britannique du XIX siècle), les grimpeurs sont incapables de saisir le "tout " irréductible qu'elle constitue. Enfin, l'allemand Caspar David Friedrich, en portant l'art romantique au $\mathrm{XIX}^{\mathrm{e}}$ siècle aux limites du fantastique, engage l'homme à la contemplation de la nature et à sa «re-création » imaginaire. L'artiste surréaliste Salvador Dalí est passé maître dans l'art de la métamorphose des reliefs qui, au sein d'un même tableau, se muent en objets ou en personnes (anthropo-zoo-morphisme, voir L'Énigme sans fin, 1938). Dans la littérature, les reliefs participent aux récits burlesques (Rabelais, Pantagruel, 1532), théâtraux et ironiques (Rostand, 1897, avec la tirade du nez de Cyrano de Bergerac) et fantastiques. 
Figure 4a. Couverture du livre Le Rayon vert, Jules Verne, L. Benett, 1881

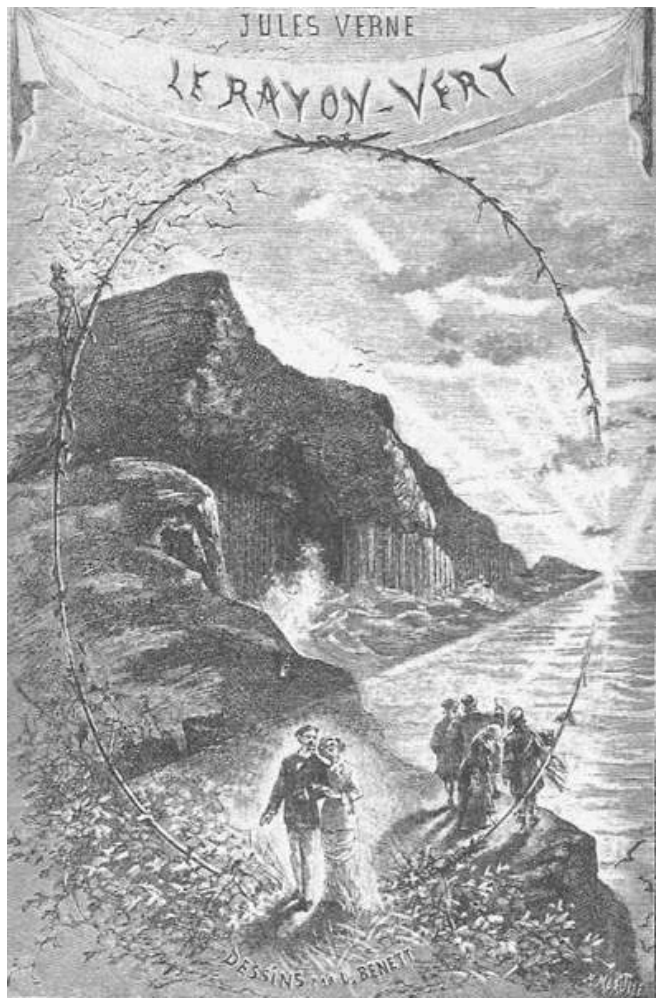

Les héros du roman cherchent à voir le rayon mystérieux en Écosse. Après de nombreuses tentatives d'observation, le phénomène se présente... Les orgues basaltiques de l'île de Staffa (ici la grotte de Fingal) constituent le décor romantique et fantastique des aventures des personnages.

Dans la science-fiction et la fantasy, romanciers, cinéastes et auteurs de bandes dessinées contemporains créent la totalité des univers et des mondes où évoluent les personnages $^{12}$. 
Figure 4b. Affiche du film Le Seigneur des anneaux, d'après le roman de John Ronald Reuel Tolkien, 1954-1955

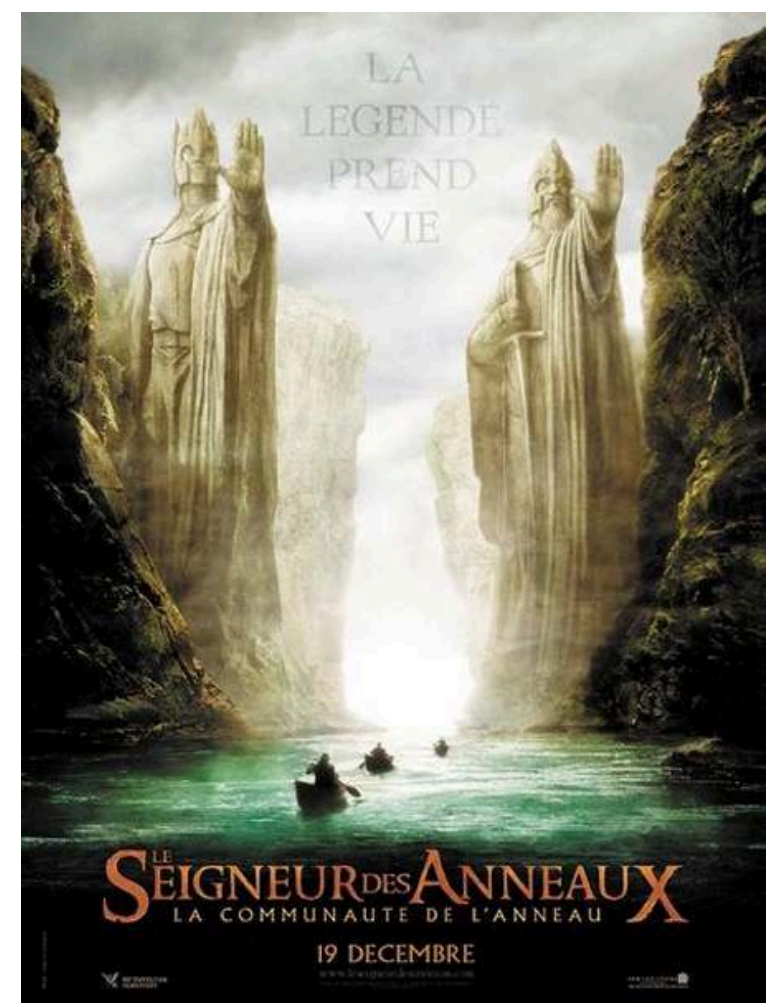

Le premier tome de la trilogie, La Communauté de l'anneau, est adapté au cinéma en 2001 (réalisateur Peter Jackson). L'affiche représente Fondcombe, lieu de la Terre du Milieu située dans une vallée encaissée aux pieds des monts Brumeux et délimitée par deux torrents.

17 L'introduction de la rationalité dans la lecture et le ressenti des paysages - et donc des reliefs - s'accentue dès le début du XIX ${ }^{\mathrm{e}}$ siècle. «Le paysage de haute montagne offre ainsi au voyageur non plus une seule scène, strictement construite pour une vision frontale, mais plusieurs « scènes continuelles » dont l'artiste doit prendre compte [...] : les aquarellistes anglais comme John Robert Cozens, Francis Towne, Joseph Wright of Derby et les peintres suisses Caspar Wolf et Heinrich Wüest décrivent avec soin les sites et en proposent déjà une typologie: la grotte, la cascade, le ravin, le glacier, les sommets, la vue panoramique. Chez Wolf, ces vues exactes contrastent avec des compositions plus sophistiquées [...]: arbres inquiétants, rochers menaçants et cascades servent de décor à des figurines tirées du répertoire rococo ${ }^{13}[. .$.$] . Cozens se$ distingue par une vision mélancolique, déjà romantique [...] (Mérot, 2009, p. 321), prémices de l'émergence du modèle pittoresque. Ainsi, par opposition à ce qui relève de l'ordre divin et surnaturel, "les faits de nature concernaient un ordre physique et matériel dont le rapport aux hommes fut marginalisé par la connaissance scientifique : à l'époque moderne, la nature [...] retrouva une dimension sensible à travers l'art des jardins et du paysage qui s'en saisit comme motif d'inspiration, en particulier au $\mathrm{XIX}^{\mathrm{e}}$ siècle. C'est ainsi que se développa, dans les arts, une intelligence sensible de la nature qui creusa l'écart avec celle qui ne passait pas par les pinceaux et le regard, mais par les concepts des sciences physiques, géographiques ou biologiques » (Donadieu, 2002, p. 24). 


\section{Du pittoresque au " pittoresque écologique »} itinérance, dans une région précise. Ils symbolisent un nouveau regard porté sur les paysages et la nature. Celle-ci, même si elle demeure un produit du divin, est moins sublimée : en relation avec l'aménagement des villes et des campagnes et avec les changements d'usages et de pratiques, la nature dans son intégralité est domestiquée. L'homme s'approprie l'espace qui devient productif et ludique dans sa ruralité, pittoresque et scientifique dans le voyage, initiatique dans les "grands tours " des classes les plus aisées de l'époque. Sur le littoral, les vertus thérapeutiques de la fraîcheur de la Manche et des vagues sont ventées (Corbin, 1988) amenant une fréquentation de ces espaces : le corps est devenu l'objet d'attention des aristocrates des $\mathrm{XVIII}^{\mathrm{e}}$ et $\mathrm{XIX}^{\mathrm{e}}$ siècle. En montagne, l'objectif est d'ouvrir de nouvelles voies. Les alpinistes s'établissent à Zermatt et le Matterhorn (le Cervin) devient le symbole d'une nouvelle idée de l'ascension : le sommet le plus haut n'est plus le but à atteindre; il faut escalader et maîtriser les pics inaccessibles. La difficulté fait place au prestige de la conquête des cimes. Alors que se construisent ces nouvelles images de la montagne et $\mathrm{du}$ littoral, la forêt de Fontainebleau est le premier espace naturel du monde à être reconnu en tant que réserve artistique (1853) avant même la création des parcs nationaux nord-américains (1872). La forêt et ses chaos de boules de grès ont été peints, photographiés et mis en poésie à de nombreuses reprises, notamment par les peintres impressionnistes de l'école de Barbizon. Les chaos et les reliefs de la forêt constituent les motifs paysagers de nombreuses productions picturales.

Figure 5. Les rochers de Fontainebleau. L'éléphant des gorges d'Apremont, forêt de Fontainebleau (Seine-et-Marne), carte postale ancienne, 1907

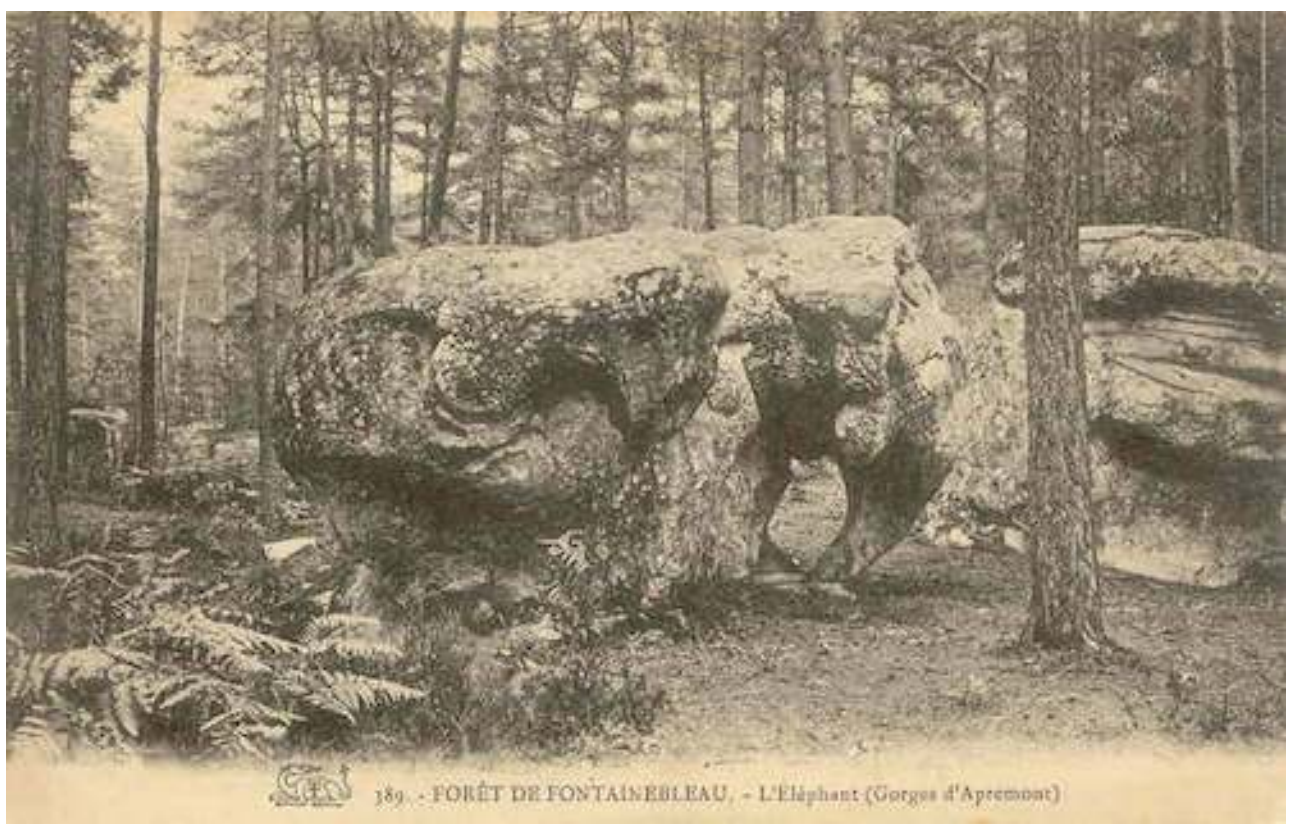

Les blocs anthropomorphiques et zoomorphiques ont dès le début du xix siècle constitué un moteur du tourisme pittoresque de la capitale.

Les affleurements rocheux, rarissimes dans le pays francilien, sont ainsi recherchés par les paysagistes pour la composition des jardins. Les reliefs font intégralement partie 
d'une organisation à l'anglaise comme en témoigne l'ouvrage d'Humphrey Repton, Théorie et Pratique de l'architecture du jardin écrit en 1816. "La délectation du jardin anglais est fondée sur une mécanique du regard. Le panorama que celui-ci se doit de parcourir résulte d'un long travail de l'architecte qui a su utiliser la morphologie du lieu, tout en l'artialisant. Au sein de ce paysage, la variété des scènes doit assurer, au fil de la déambulation, le plaisir de la vue, de l'ouïe, de l'odorat »(Corbin, 2001, p. 37). Certains reliefs sont recréés artificiellement, comme la grotte du Bosquet des Bainsd'Apollon conçue par Hubert Robert pour les jardins de Versailles.

Figure 6a. Projet pour la grotte du Bosquet des Bains-d'Apollon dans les jardins de Versailles, Hubert Robert, 1775, Versailles, châteaux de Versailles et de Trianon

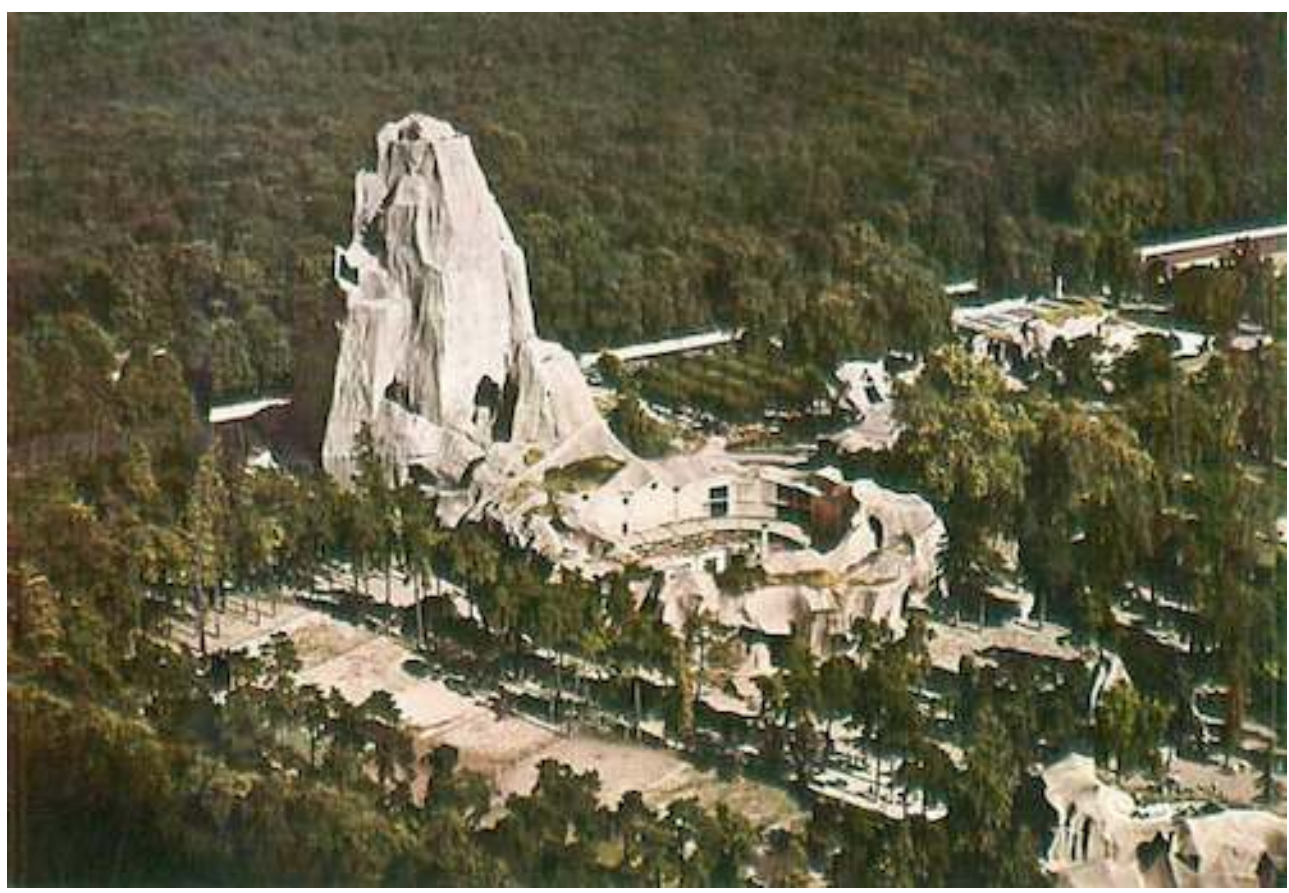

Source : http://www.culture.gouv.fr/.

En 1931, la naissance du « petit zoo de Vincennes » porte à son apogée la création de reliefs artificiels, avec la «mystique du rocher» (Pinon, 2008), reproduisant un « morceau d'Afrique » dans le contexte colonial de l'époque. 
Figure 6b. Un « morceau d'Afrique » en Île-de-France, carte postale, 1970, éditions Combier, Paris

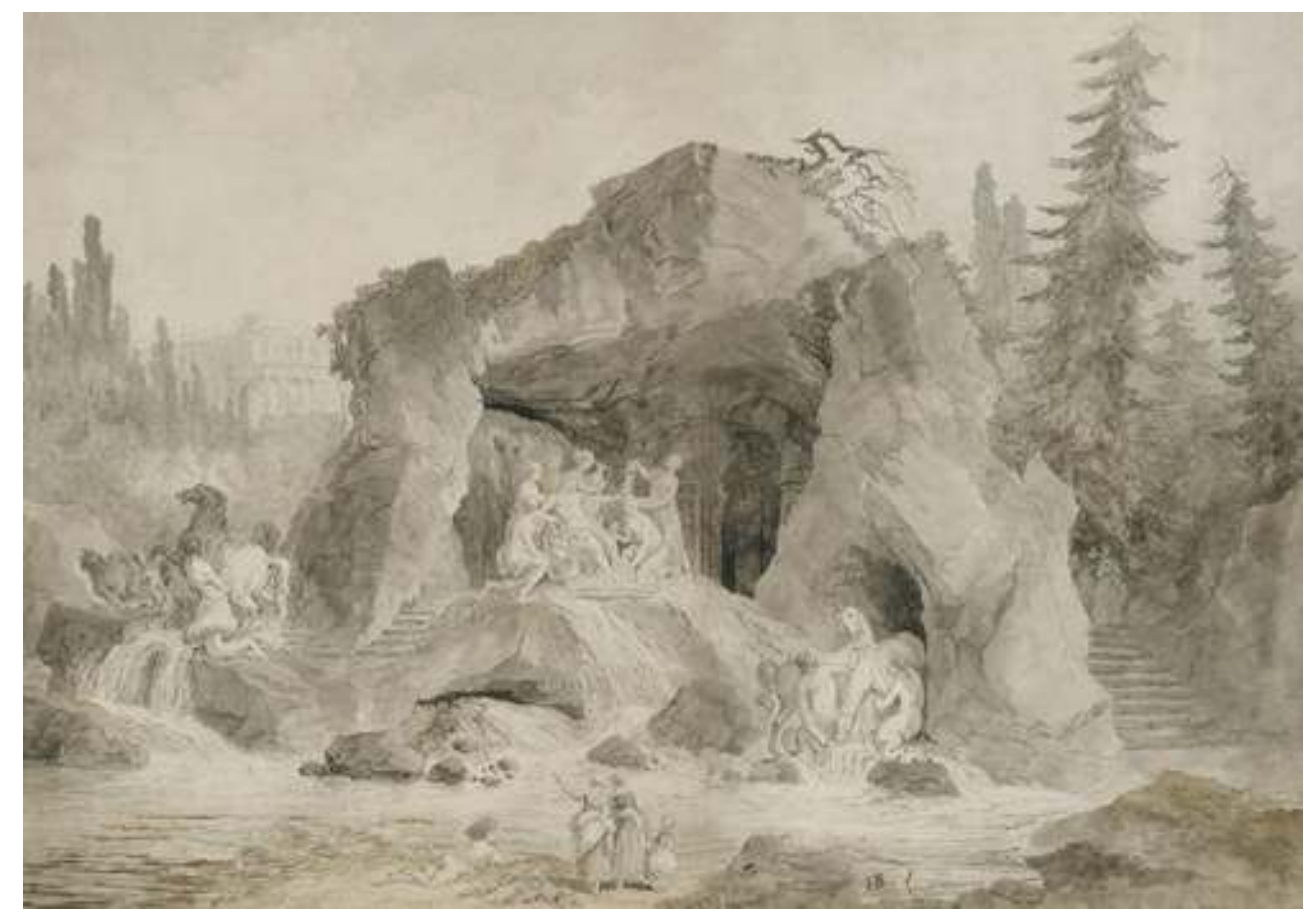

21 «Le pittoresque est sans doute lié à la formation et à la consolidation du modèle régional dans la seconde moitié $\mathrm{du} \mathrm{XIX}^{\mathrm{e}}$ siècle lorsque émerge le débat entre le national et le régional dans les milieux politiques : la formalisation du modèle régional est la suite presque naturelle des pratiques touristiques qui permettent à la bourgeoisie européenne de découvrir les singularités régionales que les écoles de géographies européennes vont ancrer dans leurs premières œuvres scientifiques » (Luginbühl, 2006, p. 65). Ce modèle paysager «pittoresque écologique » est ainsi «né de l'introduction des connaissances écologiques savantes dans les représentations sociales du paysage » (ibid., p. 83). Apparu dans les dernières décennies du $\mathrm{xx}^{\mathrm{e}}$ siècle, il croise les regards scientifiques et esthétiques. Les sciences de la terre semblent y avoir trouvé une place légitime: les paysages sont souvent géologiquement informés, notamment dans les films documentaires qui lui sont exclusivement consacrés. Le lien entre la configuration géomorphologique et la notion d'habitat écologique a été reconnu, d'abord avec les travaux des botanistes (Paul Jovet en France dans les années 1930 par exemple), puis avec la notion "d'écosystème » (Arthur George Tanslay en 1935): la valeur scientifique associée aux représentations pittoresques confère aux reliefs comme aux paysages une valeur supplémentaire qui va légitimer leur protection et participer à leur patrimonialisation.

\section{Les reliefs et les modèles académiques : la permanence des reliefs de la nation}

«Structurée par ces modèles paysagers, la culture académique se distingue des cultures «locales» qui s'élaborent par la formation de connaissances empiriques de la nature, par la mobilisation des rapports sociaux et de leurs interactions à travers les pratiques d'observation dans l'exercice des activités de la vie quotidienne » (Luginbühl, 2006, p.65). Ainsi, chaque individu structure sa pensée du paysage sur la base de trois 
échelles (locale, globale, individuelle). Un individu, une collectivité peuvent apprécier un paysage pour sa conformité avec un modèle paysager ou le rejeter parce qu'il renvoie à des significations négatives. En tant que motifs, les reliefs sont le produit d'une image de la nation à ces différentes échelles. Dans Les Figures paysagères de la nation, François Walter (2004) met en exergue la charge patrimoniale des arbres, des rochers ou des sites admirables. Les reliefs sont le support du palimpseste paysager, repères immuables et figés dans le temps, une "donnée constante de l'espace » (Le Cœur, 1987, p. 46) qui, tout comme le paysage, agissent sur la « réalité sociale ». « En retour, les sociétés ne cessent d'utiliser le vecteur paysager pour faire passer des significations, ou si l'on préfère, de la territorialité. [...] Il y a des usages politiques du paysage, une instrumentalisation des schèmes paysagers [...] parce que leur histoire a servi à [...] faire exister une expérience collective » (Walter, 2004, p. 13). Les Alpes et les montagnes européennes deviennent le siège de la construction héroïque de l'individu et de la nation (ibid.) puis des destinations prisées par les touristes des $\mathrm{XIX}^{\mathrm{e}}$ et $\mathrm{xx}^{\mathrm{e}}$ siècles. Les reliefs contribuent à l'exacerbation des sentiments héroïques, hygiénistes et romantiques et sont parfois instrumentalisés pour la construction de la «nation»: dans leur apparente immuabilité, ils constituent des éléments pérennes, repères atemporels pour la société qui peut s'y référer. Le mont Rushmore constitue à ce titre une allégorie de ce besoin de construction d'une nation à l'histoire courte autour d'un nationalisme exacerbé ${ }^{14}$.

\section{Conclusion}

«Pour les paysagistes, l'usage du mot "géographie" renvoie à l'étude de la surface de la terre, à la dynamique historique des formes naturelles et culturelles composant cette surface. Les sols, les modelés géographiques, les formes planimétriques sont étudiés dans leur interaction avec les projets de sociétés qui les ont produits et/ou modifiés » (Chomarat-Ruiz, 2010).

Ainsi, quelles que soient leurs formes, les reliefs/modelés perçus en tant que motifs participent à l'artialisation des paysages et à leur représentation. Dans certains cas, ils constituent même des "anté-motifs", soit des reliefs-mémoires dont la présence physique n'est plus visible mais dont certaines traces subsistent sur le terrain (les méandres recoupés), dans les mémoires locales (les sommets décapités des Appalaches) ou encore, dans la toponymie (les oronymes). Dans la construction du projet de paysage, reliefs visibles, mémoriels ou représentés y tiennent une place centrale (Davodeau, 2008). Ainsi, si le sens attribué au « socle géographique » par les paysagistes diffère de celui donné par les géomorphologues, il trouve cependant une complémentarité à l'interface entre regards naturaliste et culturel. Les reliefs se parent alors d'une dimension nouvelle qui les intègre comme des éléments fondamentaux motivant la « relation paysagère " (Berque, 2000, p. 48). Un dialogue, encore émergent, peut alors s'engager entre le paysagiste et le géographe, entre l'artiste et le scientifique afin de donner corps à ce socle-motif dont les dualités (nature/culture; visible/ mémoriel ; réel/imaginaire ; vécu/représenté ; permanence/mobilité) lui confèrent une dimension encore méconnue et dont la teneur se révèle palpable par une approche géographique et paysagère aux multiples regards. 


\section{BIBLIOGRAPHIE}

Aubry, P., « Motif de paysage ", dans Berque, A. (sous la dir. de), Mouvance II. Soixante-dix mots pour le paysage, Paris, Éditions de la Villette, 2006, 120 p.

Berque, A., Médiance. De milieux en paysages, Paris, Belin/Reclus, coll. « Géographiques », 2000, $160 \mathrm{p}$.

Besse, J.-M., Voir la Terre. Six essais sur le paysage et la géographie, Arles/Versailles, Actes Sud/ENSP, 2000, $161 \mathrm{p}$.

Billaut, A., « Volcanisme et esthétique : à propos du traité Du Sublime », dans Foulon, E., Connaissance et Représentations des volcans dans l'Antiquité, Clermont-Ferrand, Presses universitaires Blaise Pascal, 2004, p. 193-204

Brandon, C., Monadnock. More than a mountain, Keene, Surry Cottage Books, 2007, 416 p.

Chomarat-Ruiz C., « L'art et la science à l'épreuve du paysage, Projets de paysage [en ligne], publié le 24/02/2010, URL : http://www.projetsdepaysage.fr/fr/

1_art_et_la_science_a_l_epreuve_du_paysage.

Corbin, A., L'Homme dans le paysage. Entretien avec Jean Lebrun, Paris, Textuel, 2001, 190 p.

Corbin, A., Le Territoire du vide. L'occident et le désir de rivage, 1750-1840, Paris, Flammarion, 1988, $410 \mathrm{p}$.

Davodeau, H., « Le socle, matériau du projet de paysage. L'usage de la géographie par les étudiants de l'École du paysage de Versailles ", Projets de paysage [en ligne], publié le 30/12/2008, URL : http://www.projetsdepaysage.fr/fr/le_socle_materiau_du_projet_de_paysage.

Debarbieux, B., « Le paysage selon Frederic E. Church et la réception de Humboldt parmi les peintres américains ", Les Carnets du paysage, $n^{\circ}$ 16, 2008, p. 189-205.

Donadieu, P., La Société paysagiste, Arles/Versailles, Actes Sud/ENSP, 2002, 156 p.

Duval, M., « spatiales et enjeux territoriaux des processus de patrimonialisation et de développement touristique. Étude comparée des gorges de l'Ardèche et du karst Slovène ", thèse de géographie, université de Savoie, 2007, 516 p.

Farhat, G., «L'anamorphose du territoire », Projets de paysage [en ligne], publié le 26/12/2008, URL : http://www.projetsdepaysage.fr/fr/l_anamorphose_du_territoire.

Freytet, A., Rumelhart, M., « Une tranche de persuasion massive : le bloc-diagramme », Les Carnets du Paysage, $\mathrm{n}^{\circ}$ 20, Arles/Versailles, Actes Sud/ENSP, 2010, p. 31-42.

Freytet, A., Chazelle C., « Livret pédagogique de l'atlas des paysages de Seine-et-Marne » [en ligne], CAUE 77, conseil général de Seine-et-Marne, 2008, 205 p.

George, P., Verger, F., Dictionnaire de la géographie, Paris, PUF, 2006, 475 p.

Laneyrie-Dagen, N., L'Invention de la nature, Paris, Flammarion, 2008, 256 p.

Le Cœur, C., «Le paysage comme cadre physique ", Hérodote. Paysages en action. Le rivage des Syrtes?, n 44,1987, p. $45-50$.

Luginbühl, Y., « Modèles paysagers ", dans Berque, A. (sous la dir. de), Mouvance II. Soixante-dix mots pour le paysage, Paris, Éditions de la Villette, 2006, 120 p. 
Luginbühl, Y., Paysages. Textes et représentations du paysage du siècle des Lumières à nos jours, Lyon, La Manufacture, 1989, $270 \mathrm{p}$.

Mérot, A., Du paysage en peinture dans l'Occident moderne, Paris, Gallimard, 2009, 443 p.

Monnier, G., « Buttes, côtes et coteaux : une figure picturale de l'art moderne », Lire le paysage, lire les paysages, actes du colloque, 24-25/11/1983, université de Saint-Étienne/Centre interdisciplinaire d'études et de recherches sur l'expression contemporaine, Saint-Étienne, Cierec, 1984, p. 231-241.

Nardy, J.-P., « Réflexions sur l'évolution historique de la perception géographique du relief terrestre », L'Espace géographique, n 3, t. XI, Montpellier, Reclus, 1982, p. 224-232.

Panizza, M., Piacente, S., Geomorfologia culturale, Bologne, Pitagora ed., 2003, 350 p.

Pinon, D., « Le Zoo de Vincennes ou "la mystique du rocher" ", Polia, revue de l'art des jardins, n 9 , Sully-la-Tour, Findakly, 2008, p. 53-82.

Portal, C., "Reliefs et patrimoine géomorphologique. Applications aux parcs naturels de la façade atlantique européenne", thèse de doctorat, université de Nantes, 2010, 436 p., URL : : http:// tel.archives-ouvertes.fr/tel-00537350/fr/.

Repton, H., Fragments on the Theory and Practice of Landscape Gardening. Including some remarks on Grecian and Gothic Architecture, London, T. Bensley et Son for J. Taylor at the architectural library, 1816, 349 p.

Rey, A. (sous la dir. de), Dictionnaire historique en langue française, Paris, Le Robert, t. II, 1992, $2400 \mathrm{p}$.

Roger, A., " Histoire d'une passion théorique ou comment on devient un Raboliot du Paysage », dans Roger, A. (sous la dir. de), La Théorie du paysage en France (1974-1994), Seyssel, Champ Vallon, 1995, p. 438-453.

Rumelhart, M., « Vous avez dit « géomorphologie » ? ", Les Carnets du paysage, nº 5, Arles/ Versailles, Actes sud/ENSP, 2000, p. 167-172.

Schama, S., Le Paysage et la Mémoire, Paris, Seuil, 1999, 722 p.

Walter F., Les Figures paysagères de la nation : territoire et paysage en Europe ( $\mathrm{XVI}^{e}-\mathrm{XX}^{e}$ siècle), Paris, École des hautes études en sciences sociales, 2004, 528 p.

\section{NOTES}

1. Un relief est une saillie à la surface du globe (colline ou montagne). L'importance d'un relief se mesure à partir de son étendue et à sa hauteur appréciée à la verticale à partir de sa base. Un relief d'érosion, issu du dégagement de matériaux - "un relief en creux» -, ou d'une accumulation (plage, dune) est appelé modelé (George et Verger, 2006, p. 304).

2. Pour les géomorphologues, le socle est un «ensemble des terrains indurés, c'est-à-dire fortement métamorphisés et granitisés, où se juxtaposent parfois des éléments formés au cours d'orogène successif (George et Verger, 2006, p. 398). Un socle est toujours ancien, et est constitué de matériaux qui ont été remaniés par des mouvements tectoniques. Le socle constitue donc une base (basement/bedrock) qui peut supporter d'autres roches (sédimentaires par exemple) et l'ensemble paysager qui s'offre à la vue.

3. Voir entre autres - et à titre d'exemples - Les Essais de Montaigne (1580), Waterloo de Victor Hugo (1853) [« Waterloo! Waterloo! Waterloo! Morne plaine! Comme une onde qui bout dans 
une urne trop pleine, Dans ton cirque de bois, de coteaux, de vallons, La pâle mort mêlait les sombres bataillons [...]») ou encore Romance sans paroles de Paul Verlaine (1874) («Dans l'interminable Ennui de la plaine, La neige incertaine, Luit comme du sable...»].

4. Voir http://tel.archives-ouvertes.fr/tel-00537350/fr/, p. 73.

5. Voir entre autres Léonard de Vinci et ses Études de formations rocheuses (Schama, 1999).

6. Le volcan structure physiquement le paysage et devient l'objet unique de toutes les attentions littéraires au XIX ${ }^{\mathrm{e}}$ siècle (Billaut, 2004). À cette époque, le mont Fuji est le sujet de nombreuses représentations (Les Trente-Six Vues du mont Fuji d'Hokusai Katsushika, 1830-1832) en tant que montagne sacrée. Aux États-Unis, le parc national de Yellowstone a été créé en 1872 en raison de ses phénomènes géothermiques (geysers, sources chaudes).

7. Le terme «modelé » est aussi un terme de sculpture : tout comme le sculpteur taille un bloc de marbre pour lui donner une forme, l'érosion apporte des touches et des retouches à partir de masses mises en place par la tectonique ou par des processus d'érosions antérieurs.

8. Voir http://tel.archives-ouvertes.fr/tel-00537350/fr/, p. 79.

9. Nous nuançons ici notre propos : il y a toujours une morphogenèse ralentie en période de biostasie. Hormis lors de phénomènes tectoniques et volcaniques, et perçues à l'échelle humaine, les grandes formes de reliefs semblent cependant immobiles et immuables.

10. L'allégorie de la caverne est exposée par Platon dans le Livre VII de La République. Elle met en scène des hommes enchaînés et immobilisés dans une "demeure " souterraine. Ces hommes tournent le dos à l'entrée et ne voient que leurs ombres et celles d'objets, projetées au loin derrière eux. Cette allégorie expose métaphoriquement l'accession des hommes à la connaissance de la réalité, ainsi que la transmission de cette connaissance.

11. Cette découverte du paysage intérieur n'est cependant pas issue du XIXe siècle. En 1336, Pétrarque ascensionne le mont Ventoux, emportant avec lui l'œuvre de Saint-Augustin. Pour le poète italien, le paysage est une "passion de l'extérieur ", une "sortie dans l'étendue ", une « sorte de maladie » (Besse, 2000).

12. Les représentations imaginaires des reliefs sont aussi anciennes: la Carte du pays de Tendre, représentation cartographique de la relation amoureuse vue par les précieuses du XVII siècle, est charpentée aux quatre points cardinaux par des affleurements rocheux ; celui en bas à gauche est un piton rocheux nommé « orgueil».

13. L'expression rococo vient de la contraction de rocaille et de baroque.

14. Le mont Rushmore (Dakota du Sud) est une sculpture monumentale en granit localisée à l'intérieur du mémorial présidentiel des États-Unis qui retrace 150 ans de l'histoire du pays. Les sculptures, hautes de 18 mètres, représentent quatre des 44 présidents de l'histoire des ÉtatsUnis. Le mémorial couvre une surface de $5,17 \mathrm{~km}^{2}$ et se situe à 1745 mètres d'altitude. Il est géré par le National Park Service qui dépend du département de l'Intérieur des États-Unis et attire plus de deux millions de visiteurs chaque année.

\section{RÉSUMÉS}

Cet article étudie la composante géomorphologique des paysages artialisés. Les reliefs sont ici considérés comme des motifs de paysages constituant le «socle géographique » du paysagiste, revêtus d'un aspect culturel émanant d'un nouveau champ de recherche, la géomorphologie culturelle. En tant que motifs, ils participent ainsi à la composition picturale et lyrique d'œuvres 
artistiques et se révèlent comme des éléments moteurs de la construction des modèles paysagers occidentaux.

This paper studies the geomorphic component of "landscapes artialisation" by studying the major landforms in landscapes' representations. Landforms are considered here as landscapes patterns constituting the "geographical basement " for landscape painter, holding a cultural aspect emanating from a new field of research, cultural geomorphology. As patterns, landforms also participate in the pictorial and lyric composition of artistic works and show themselves as driving elements of the construction of western landscapes models.

INDEX

Keywords : landform pattern, cultural geomorphology, landscape model, bedrock, geography

Mots-clés : relief-motif, géomorphologie culturelle, modèle paysager, socle, géographie

\section{AUTEUR}

\section{CLAIRE PORTAL}

Géographe, elle est postdoctorante au Laboratoire Géolittomer - UMR LETG 6554 - CNRS.

claire.portal[at]univ-nantes[dot]fr 